\title{
The left common carotid artery rises from the main pulmonary artery
}

\author{
Vu Tuan Nguyen* \\ Faculty of Medicine PNT HCMC, Medic HCMC Vietnam
}

\begin{abstract}
A young female patient of $15 y$.o presented at my hospital by dyspnea on effort and palpitation for one year. Mental deficiency was notified. Physical examination detected a 3/6 continuous murmur at the 2ndRICS. In the past history, PDA had been suspected by her physician, associated with recurrent bronchitis.

Trans-thoracic Echocardiography showed an enlarged LV of $57 \mathrm{~mm}$ with normal EF of $69 \%$, LCA=5mm, RCA=3.5mm at origin, no suspected sign of PDA was seen. Only a continuous flow was visualized in the PA.

CT-Angiography with IV contrast medium showed the Left Common Carotid Artery rising from the Pulmonary Artery trunk. PDA was not presented.

The Left Common Carotid Artery then was re-implanted into the aortic arch normally with a favourable post-operative progress.
\end{abstract}

\section{Introduction}

Anomalous origin of the left common carotid artery is very rare and has been reported previously. We present an operated case of this topic with clinical finding, cardiac ultrasound and MDCT imaging.

\section{Case report}

A young female patient of $15 y$.o presented at my hospital by dyspnea and palpitation when running and fast walking for one year. Mental deficiency was notified, she had some difficulties to learn at school. Physical examination detected a $3 / 6$ continuous murmur at the 2ndRICS. In the past history, PDA has been suspected by her physician, associated with recurrent bronchitis. Her body state was normal with $1 \mathrm{~m} 60$ of height and $48 \mathrm{~kg}$ of weight.

She was evaluated immediately by a chest $\mathrm{X}$ ray that showed a right aortic arch (Figure 1).

The trans-thoracic echocardiography that revealed an enlarged LV of $57 \mathrm{~mm}$ with normal $\mathrm{EF}$ of $69 \%$ (Figure 2), $\mathrm{LCA}=5 \mathrm{~mm}, \mathrm{RCA}=3.5 \mathrm{~mm}$ at origin (Figure 3).

No suspected sign of PDA was detected except a continuous flow presented in the Pulmonary Artery (Figure 4).

CT-Angiography (MDCT 64) with IV contrast medium Ultravist, slice thickness $=1 \mathrm{~mm}$ visualized a right aortic arch, aberrant origin of the left subclavian artery, dilatation of the branches rising from aortic arch with increased collateral vessels (Figure 5).

Especially, MDCT 64 showed the Left Common Carotid Artery (LCCA) rose from the PA trunk (Figure 6). PDA was not detected.

Patient underwent uncomplicated surgical repair: the Left Common Carotid Artery was re-implanted into the aortic arch normally with a favorable post-operative progress [1] (Figure 7).

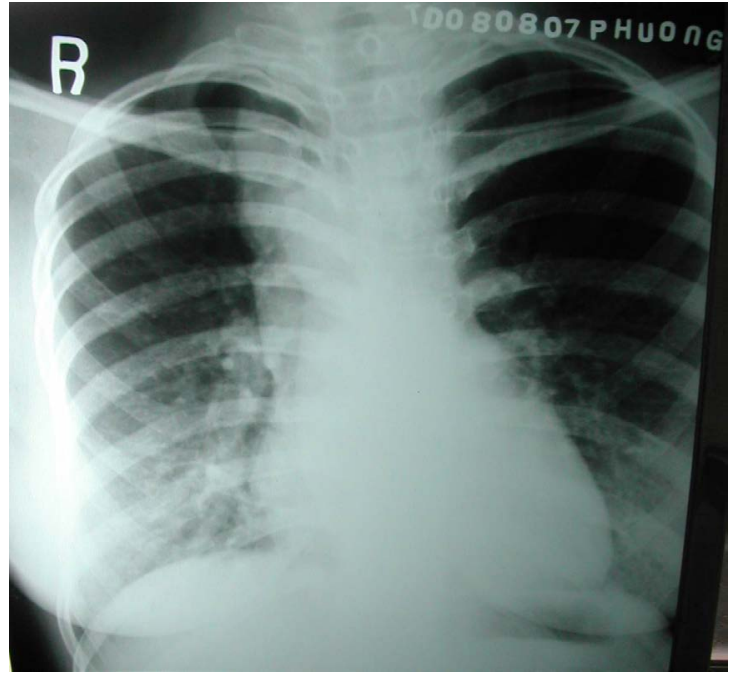

Figure 1. Right aortic Arch

\section{Discussion}

Anomalous origin of the Left Common Carotid Artery from the Pulmonary Artery Trunk has been previously reported as rare cases.

Kagami Mijaji has reported a case of anomalous origin of the Innominate Artery from the Right Pulmonary Artery [2].

${ }^{\star}$ Correspondence to: Nguyen VT, Faculty of Medicine PNT HCMC, Medic HCMC Vietnam; Email: tuanvu2401@gmail.com

Key words: carotid artery, pulmonary artery, anomalous origin

Received: June 23, 2019; Accepted: July 01, 2019; Published: July 04, 2019 


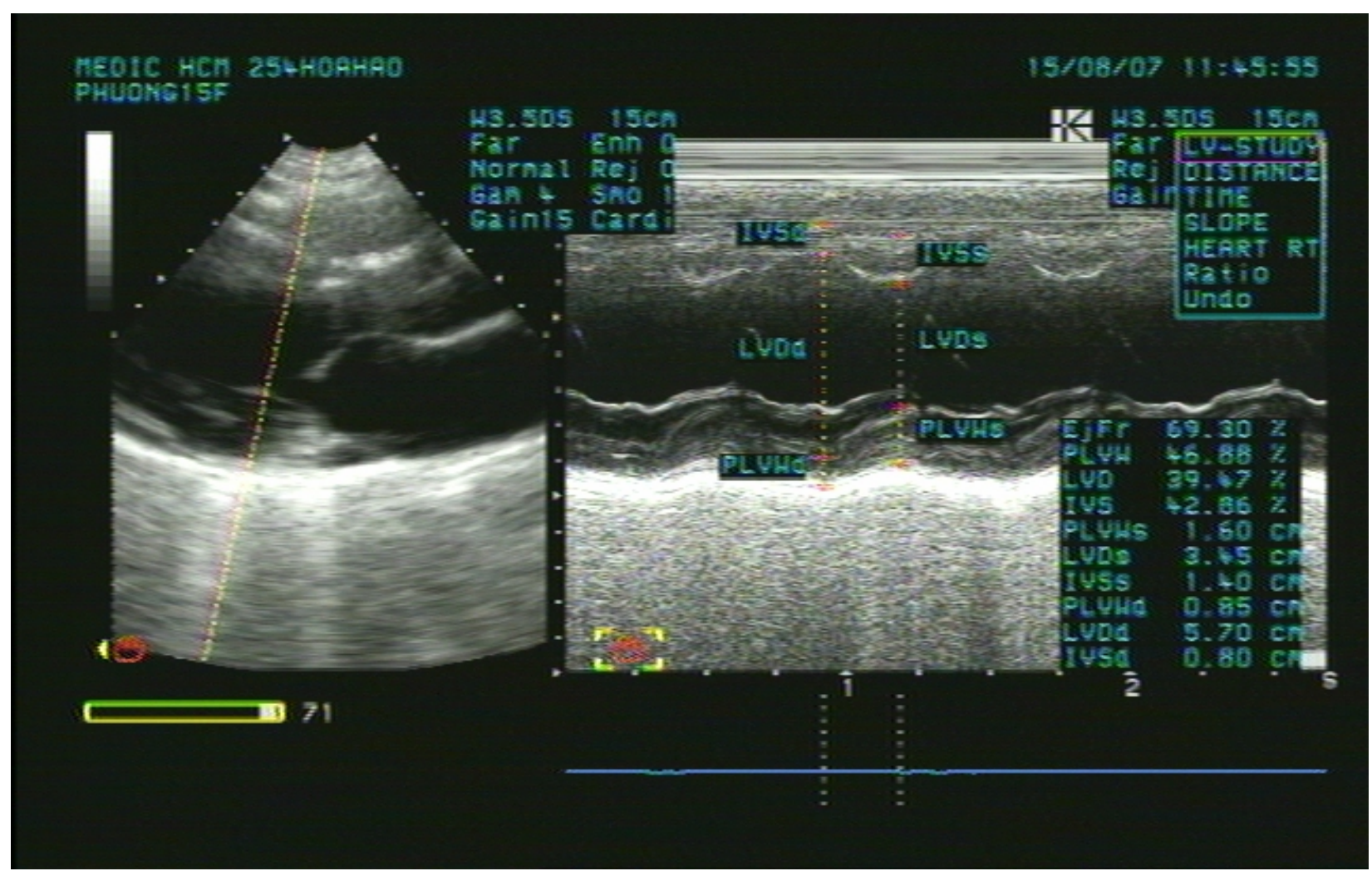

Figure 2. Enlarged LV\& normal systolic function

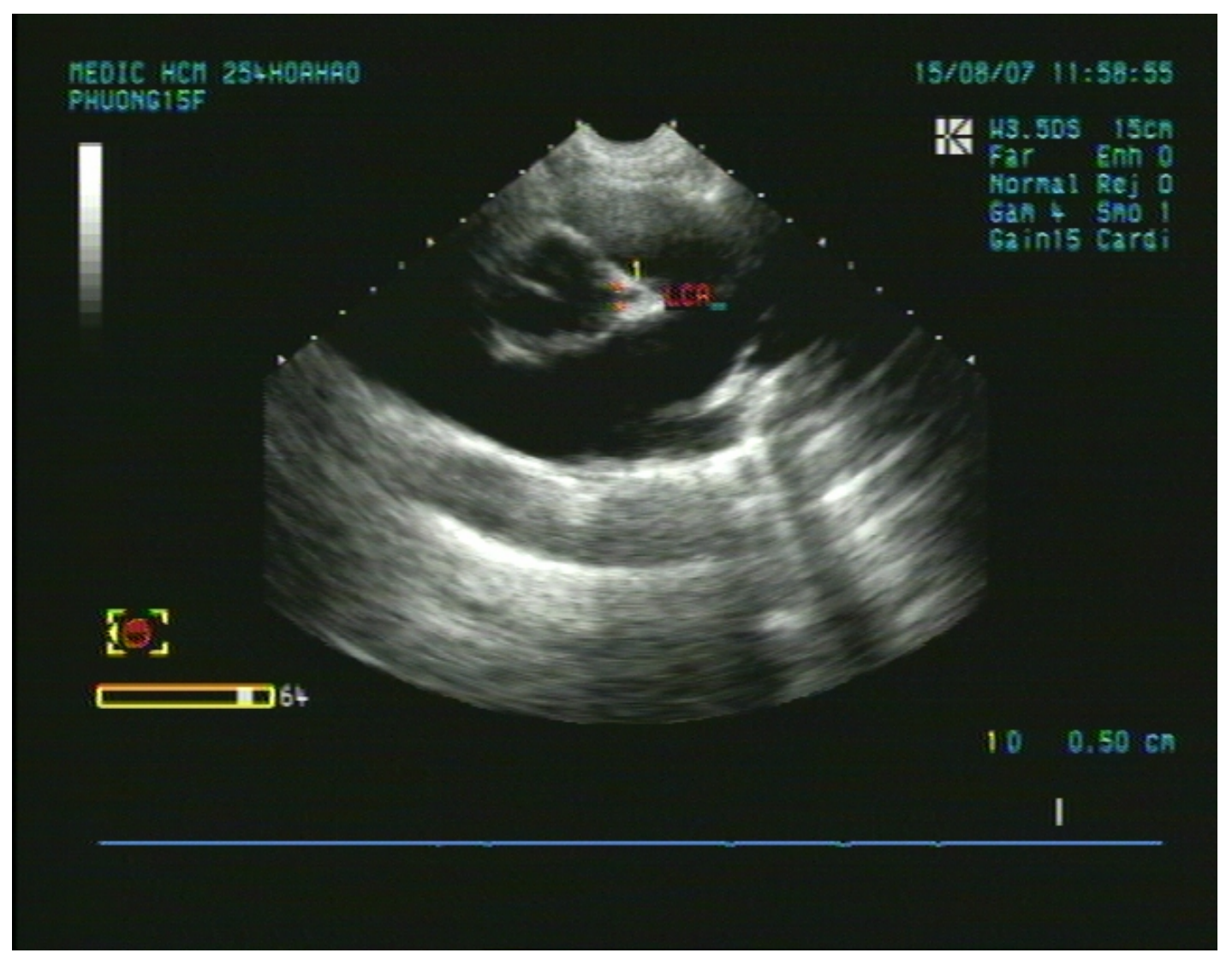

Figure 3. Normal LCA at origin 
REOTC HCH 25+HOAHAD

\section{PFuaksis}

$0.60 n / s$

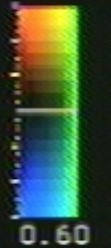

\section{$15 / 08 / 07$ 12:03:45}

H

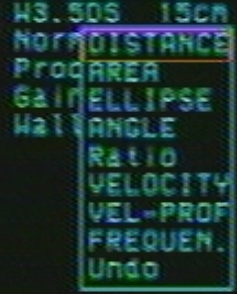

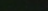

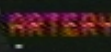
Undo

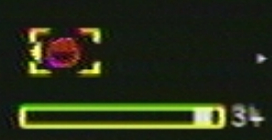

Figure 4. Continuous flow in the PA

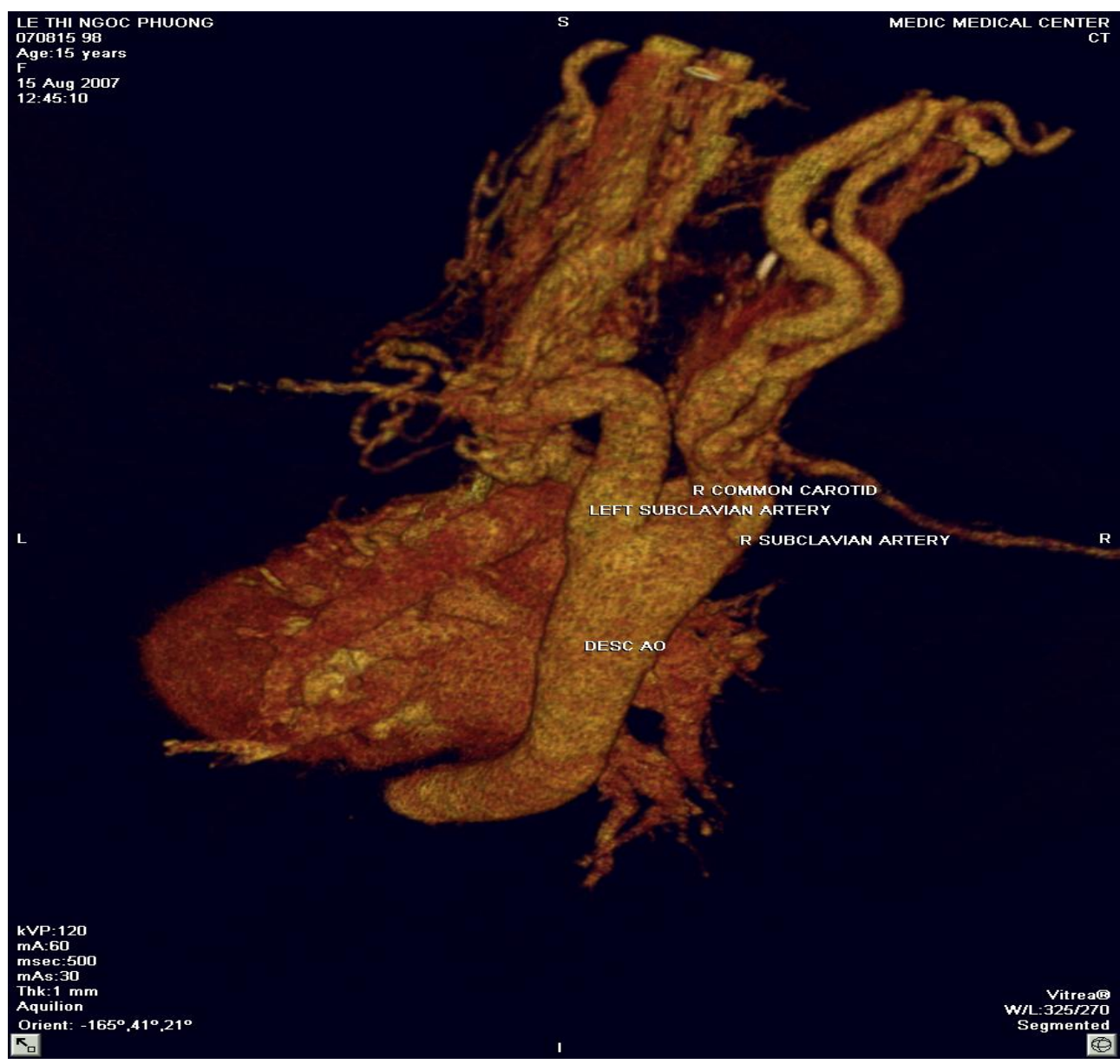

Figure 5. Absence of aortic origin of the LCCA 


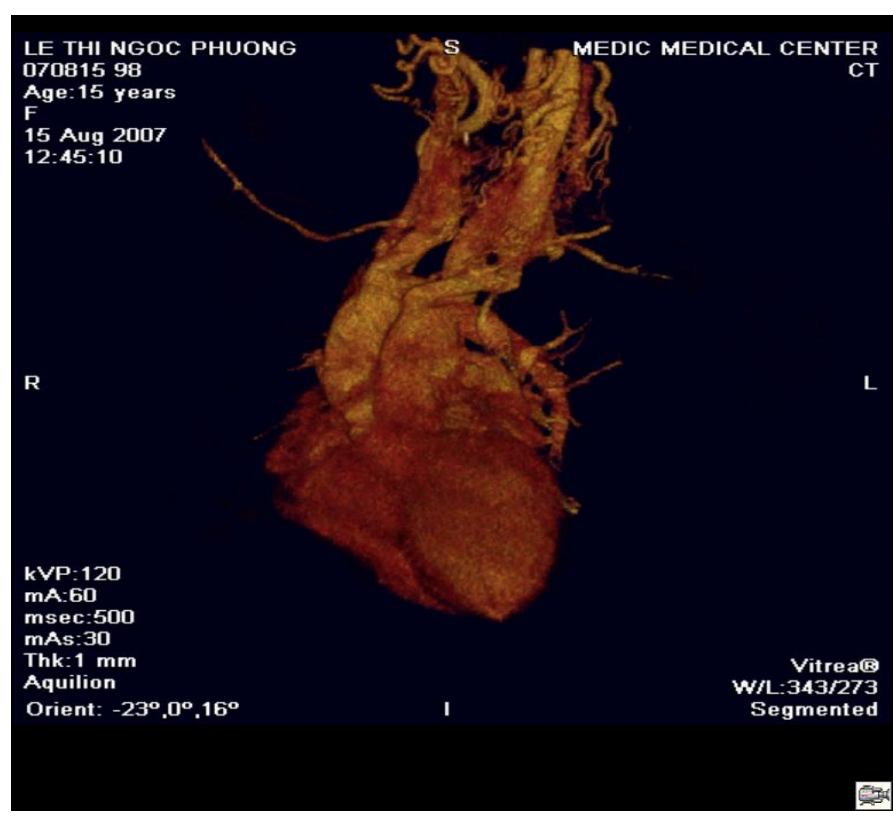

Figure 6. The LCCA rising from the Main PA roof

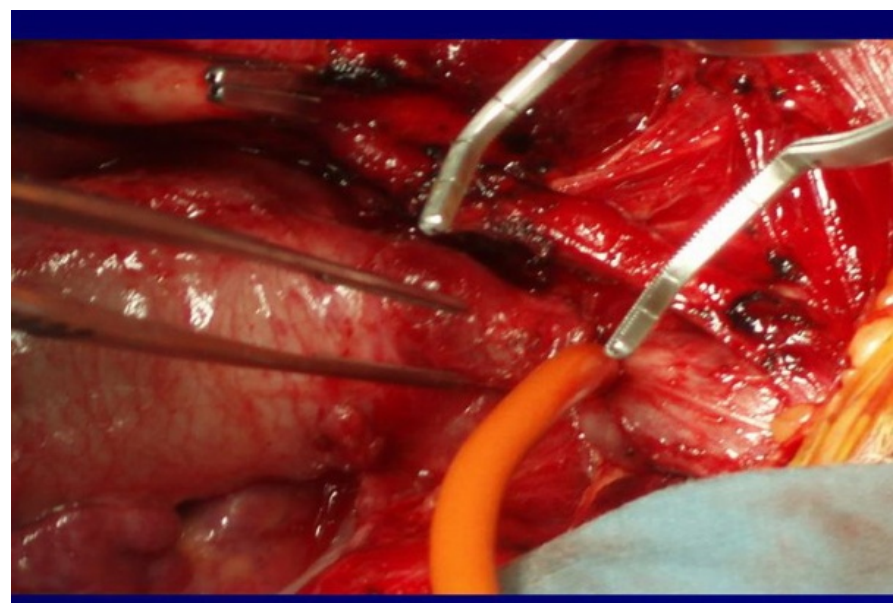

Figure 7. Re-implantation of the LCCA
Onyekachukwu, et al. has described a case of anomalous origin of the Left Common Carotid Artery from the Main Pulmonary Artery [3].

Other reports related to the anomalous origin of the Carotid Artery or the Vertebral Artery from a vessel in the systemic circulation like the brachiocephalic trunk.

In this article, my patient was not infant with CHARGES syndrome that includes multiple congenital anomalies like the patients in their reports. She was a teenage patient without other congenital disease.

The role of ultrasound is orienting for the indication of Computed Tomography or DSA. In case of present turbulent flow in the PA, Coronary Fistula and other shunts from the head and neck vessels should be considered.

\section{Conclusion}

Anomalous origin of the Left Common Carotid Artery is very rare congenital defect that maybe isolated or associated with some syndromes.

Non-invasive diagnostic methods as Ultrasound and CTA may confirm the diagnosis and inform the anatomical relation of the anomalous vessels prior to operate.

\section{References}

1. Hoffmann MHK (2005) Coronary Angiography with Multislice Computed Tomography. JAMA 293: 2371- 2478.

2. Miyaji K (2001) Anomalous origin of innominate artery from right pulmonary artery in DiGeorge syndrome. Ann Thorac Surg 71: 2043-2044.

3. Osakwe O, Jones B, Hirsch R (2016) Anomalous Origin of the Left Common Carotid Artery from the Main Pulmonary Artery: A Rare Association in an Infant with CHARGE Syndrome. Case Reports in Pediatrics.

Copyright: (C2019 Vu Tuan Nguyen. This is an open-access article distributed under the terms of the Creative Commons Attribution License, which permits unrestricted use, distribution, and reproduction in any medium, provided the original author and source are credited. 